\title{
Extensão de Cobertura ou Reorganização da Atenção Básica? A trajetória do Programa de Saúde da Família de Manaus-AM'
}

\author{
Coverage Extension or Reorganization of Primary Health \\ Care? The Implementation of the Family Health Program in \\ the City of Manaus, State of Amazonas
}

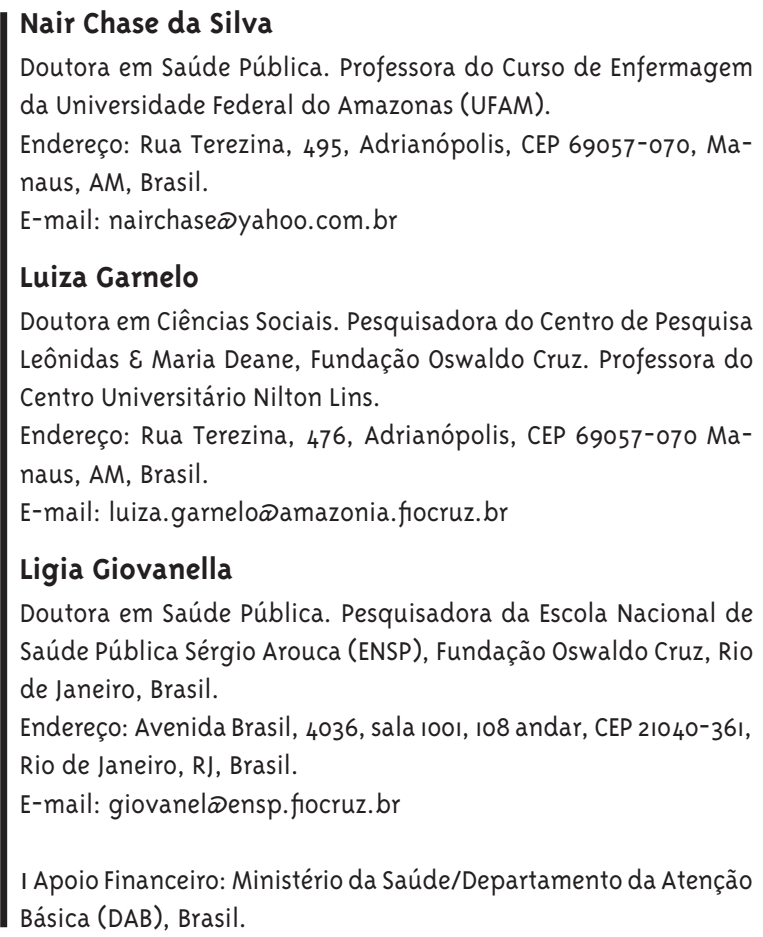

\section{Resumo}

Este trabalho analisa sete anos de implantação do Programa de Saúde da Família (PSF) de Manaus, procurando identificar mudanças no sistema municipal de saúde a ele relacionadas e capazes de contribuir para a redução de desigualdades em saúde. Contextualiza a gênese do PSF no município, suas características, contradições e limitações, investigando se o programa construiu apenas uma trajetória de extensão de cobertura ou contribuiu efetivamente para a reorganização do modelo de atenção básica à saúde. As categorias de análise priorizaram princípios específicos de gestão do PSF: caráter substitutivo, integração com instituições e organizações sociais, territorialização, planejamento situacional com base na família e na comunidade, participação popular e controle social. A metodologia utilizada foi quali-quantitativa, compreendendo a análise dos dados de duas pesquisas avaliativas da implementação do PSF no município, realizadas em 2001 e em 2006. Os resultados mostram que, em Manaus, o PSF se constitui em uma estratégia de extensão de cobertura, com parcial superposição à estrutura assistencial de atenção básica (AB) preexistente e paralelismo de ações. Dadas as condições de sua implantação, conclui-se que o PSF se expressa como um programa isolado dentro do sistema municipal de saúde, com potencialidade para se converter em estratégia reestruturadora da $\mathrm{AB}$ em Manaus. A confluência com a implantação dos Distritos Sanitários poderá contribuir para um caminho de reorientação do modelo assistencial que garanta atenção integral e concretize o direito à saúde.

Palavras-chave: Atenção Primária à Saúde; Programa de Saúde da Família; Gestão em Saúde. 


\section{Abstract}

This paper analyzes seven years of implementation of Programa Saúde da Família (PSF - Family Health Program) in the city of Manaus, state of Amazonas, aiming at identifying its influence on changes in the municipal health care system that are capable of contributing to the reduction in health care inequalities. It contextualizes the genesis of the PSF in the municipality, its characteristics, contradictions and limitations, thus investigating whether the program has built only a coverage extension history or whether it has effectively contributed to the reorganization of the primary health care model. The analysis categories prioritized specific management principles of the PSF: substitutive nature, integration with institutions and social organizations, territorialization, situational planning based on the family and community, popular participation and social control. The methodology was qualitative-quantitative, including the analysis of data from two studies evaluating the implementation of the PSF in the municipality, carried out in 2001 and 2006. The results show that, in Manaus, the PSF is a coverage extension strategy, with partial superposition on the preexisting primary medical care structure and parallelism of actions. Given the conditions of its implementation we conclude that the PSF expresses itself as an isolated program within the municipal health care system, with the potentiality to become a restructuring strategy of primary health care in Manaus. The confluence with the implementation of the Sanitary Districts may be able to contribute to redirect the medical attention model, thus assuring integral care and concretizing the right to health.

Keywords: Primary Health Care; Family Health Program; Health Management.

\section{Introdução}

A Estratégia Saúde da Família tem, entre suas origens, o movimento da medicina familiar que surgiu na década de 1960 nos EUA, em contraposição às ideias hegemônicas no ensino médico da época. Essa proposta migrou para a América Latina, inclusive para o Brasil, onde se estruturou em um contexto diferenciado. Aqui não teve como foco uma oposição ao modelo de formação médica, mas a busca de uma prática generalista e humanizada, capaz de contemplar as ideias de determinação social das doenças. No entanto, o movimento da reforma sanitária brasileira, que buscava tornar hegemônica a ideia de saúde como direito de cidadania (Teixeira, 2003/2004; Trad e Bastos, 1998; Garbois e col., 2008; Conill, 2008), tornou o acesso aos serviços de saúde uma prioridade das políticas públicas. No Brasil, a promoção do acesso ao sistema de saúde foi implementada através da rede de cuidados primários de saúde implantada em todo o território nacional (Escorel e col., 2007).

Segundo a Organização Pan-americana de Saúde (OPS) e a Organização Mundial de Saúde (OMS) (2005), a Atenção Primária à Saúde (APS) tanto pode ser entendida como seletiva, isto é, um programa dirigido a populações e regiões pobres como um primeiro nível da assistência, ou seja, como porta de entrada do sistema; e ainda como estratégia de reorganização do sistema de saúde e como um tema de direitos humanos. Tais concepções não são excludentes e, não raro, coexistem entre si (Mendes, 2002; OPAS/OMS, 2005). No Brasil, a implementação da APS se fez, principalmente, através da chamada Atenção Básica (AB) à Saúde, a qual guarda características da concepção seletiva de APS, mas com reconhecida potencialidade para reorganizar o sistema de serviços de saúde rumo a uma operacionalização abrangente de APS (Mendes, 2002; Giovanella, 2008).

É nesse contexto que o Programa de Saúde da Família (PSF) emergiu inicialmente como um programa vertical, induzido pelo Ministério da Saúde (MS) mediante a disponibilização de incentivos financeiros aos municípios que o adotassem (Soares, 200o). Como programa seletivo, o PSF teve como finalidade promover a extensão de cobertura às populações 
desassistidas, ganhando visibilidade no cenário nacional (Escorel e col., 2007; Conill, 2008). Num segundo momento, o PSF foi alçado à condição de estratégia para reorganização do sistema de saúde no âmbito municipal, e de transformação do modelo assistencial hegemônico na atenção ambulatorial do Sistema Único de Saúde (SUS), caracterizado pela atenção programática e à demanda espontânea.

A adoção de novas práticas na ESF visa ultrapassar o enfoque seletivo da $\mathrm{AB}$ rumo à abordagem integral das dimensões biopsicossociocultural e relacional da população atendida e à construção de uma rede regionalizada e hierarquizada de serviços de saúde em espaços territoriais específicos com vistas a promover também a intersetorialidade.

A implantação do PSF no Brasil está cercada de controvérsias, havendo posições favoráveis e contrárias a ela (Trad e Bastos, 1998; Garbois e col., 2008; Viana e Dal Poz, 2005; Teixeira, 2003; Merhy e Franco, 2002). Críticos da proposta (Merhy e Franco, 2002; Franco e Merhy, 2003) são contundentes ao afirmar as limitações da proposta ministerial de reordenadora, através da ESF, do modelo hegemônico de atenção na $A B$. Esses autores argumentam que a medicina comunitária, os cuidados primários de saúde e o PSF partilham propósitos de redução de custos e de simplificar a atenção à saúde ofertada aos grupos de baixa renda. Para eles, situações cotidianas, tais como a escuta qualificada das queixas, o acolhimento e o estabelecimento de vínculos entre os serviços/profissionais e a população não podem ser supridos nem pela clínica nem pela epidemiologia ou pela vigilância à saúde; e no dia a dia dos serviços tais ações permanecerão restritas ao senso comum das equipes multiprofissionais, que não dispõem de um modelo teórico que as oriente. Tais características inviabilizariam a desejada conversão do modelo assistencial por meio do PSF. Igualmente há críticas (Teixeira, 2003/2004; Merhy e Franco, 2002; Franco e Merhy, 2003) quanto à busca de um modelo padrão, nos moldes das chamadas ações programáticas, para um país tão diversificado quanto o Brasil. Tal opção promoveria a desvalorização das experiências locais, penalizadas por repasses financeiros inferiores àqueles concedidos aos que optaram pelo modelo consagrado pelo MS (Campos, 2008).

Os autores que avaliam positivamente o PSF en- fatizam sua importância para a extensão de cobertura a populações desassistidas (Teixeira, 2003/2004; Souza, 2002; Rosa e Labate, 2005); sua contribuição para a reorganização de sistemas locais de saúde e para a reversão atual do modelo hegemônico de assistência. Porém, também ressalvam que a expansão de cobertura é relevante, mas insuficiente para propiciar a reorientação do modelo (Teixeira, 2003/2004; Souza, 2002).

Em Manaus, pesquisas realizadas em 2002 (Escorel e col., 2002) e 2006 (Mendonça e col., 2006) mostram que a trajetória percorrida pelo PSF tanto se reflete nas contradições presentes no cenário nacional, quanto no contexto político e organizacional local, que configurou sua criação. Aprovado no ano de 1998 em reunião extraordinária do Conselho Municipal de Saúde de Manaus, a implantação do programa só ocorreu em 1999. Nessa ocasião, deuse uma mudança de rumos no processo de gestão do programa, que, à revelia do Conselho Municipal de Saúde, foi implementado como Programa Médico da Família (PMF). A exemplo das demais cidades, Manaus seguiu a tendência de instalar as equipes de saúde da família em áreas onde não existiam serviços de saúde, ou cujo acesso era precário, com alto risco sanitário, em processo de expansão demográfica e com populações de baixo poder aquisitivo.

Decorridos sete anos de implantação, é pertinente buscar respostas para a pergunta ora formulada: Em Manaus, o PSF construiu apenas uma trajetória de extensão de cobertura ou contribuiu para a reorganização do modelo assistencial na AB? Se o fez, de que modo isso ocorreu? $\mathrm{Na}$ busca de respostas a tais questões elegeram-se como categorias de análise os princípios específicos de gestão do PSF: a) caráter substitutivo, ou seja, se o PSF foi capaz de induzir mudanças no processo de trabalho nas Unidades Básicas de Saúde (UBS) já implantadas, redirecionando-o segundo os princípios da vigilância à saúde, promovendo a adscrição de clientela, diagnóstico situacional de saúde da população e planejamento baseado na realidade local; b) territorialização, entendida como a demarcação de um espaço social circunvizinho às unidades de saúde, estabelecendo responsabilidade sanitária para com a população ali residente; $c$ ) planejamento situacional com base na família e na comunidade, descentralizado e par- 
ticipativo, que subsidie a programação das ações de saúde no território; $d$ ) integração com instituições e organizações sociais, seja no âmbito intersetorial, seja na articulação com rede de maior complexidade; e) participação popular e controle social das ações e serviços de saúde (Brasil, 1997, 2003, 2006; Sousa e Haman, 2009).

Nessa perspectiva, este artigo tem como objetivos estudar as características de implantação e operacionalização do PSF em Manaus e analisar seus avanços e limites, além do potencial de contribuição para substituir práticas convencionais de assistência à saúde na $\mathrm{AB}$ na cidade.

\section{Metodologia}

Este artigo analisa parte dos dados coletados em duas pesquisas vinculadas ao Projeto de Expansão e Consolidação da Saúde da Família em Grandes Centros Urbanos (PROESF), do Ministério da Saúde. Na primeira, realizada em 2002, buscou-se avaliar o processo de implantação do PSF, analisar as características do programa em seus primeiros anos de implementação em Manaus e os fatores que facilitavam ou dificultavam a proposta de mudança do modelo da atenção e de reorganização do sistema municipal de saúde, para o período de 1999 a 2002 (Escorel e col., 2002).

$\mathrm{Na}$ pesquisa de 2002, dados foram coletados mediante entrevistas com o secretário municipal de saúde, com o coordenador municipal do PMF/PSF, com gerentes de programas de saúde e conselheiros de saúde. 0 roteiro de entrevista compreendeu informações sobre o processo de implantação do PMF/PSF, organização e gestão, integração à rede assistencial e conversão do modelo de $\mathrm{AB}$, vínculo empregatício e capacitação de recursos humanos, fatores facilitadores e limitadores à implantação e desempenho do PMF/ PSF e exercício do controle social. Para profissionais das Equipes de Saúde da Família (ESF), aplicou-se questionário fechado estruturado em cinco tópicos: inserção e capacitação no PSF, trabalho em equipe, integração do PSF à rede de serviços de saúde, vín- culos com a comunidade e avaliação livre dos pontos positivos e negativos do PSF.

A segunda pesquisa, realizada entre 2004 e 2006, teve como objetivo estabelecer uma linha de base do PSF com vistas à implantação de um sistema de monitoramento e avaliação no âmbito da atenção básica no país (Mendonça e col., 2006). A investigação levantou dados referentes à operacionalização do PSF em Manaus, no período de 1999 a 2006. Dentre as fontes de dados secundários foram priorizados os documentos oficiais do sistema municipal de saúde, como planos de saúde, relatórios de gestão, manual do PSF de Manaus e relatórios do PMF/PSF, disponibilizados pela Secretaria de Saúde do município, para os anos 1999 a $2006^{2}$. A coleta e análise documental foram orientadas por um resumo analítico no qual se buscou sintetizar informações referentes aos indicadores de saúde, número de profissionais, instrumentos de planejamento, estrutura da atenção básica no município, ações programáticas, prioridades da agenda municipal de saúde e planos e metas para o período. Igualmente foi investigada a coerência entre o plano municipal de saúde e os relatórios de gestão para o período correspondente, além da utilização desses documentos como instrumentos para o controle social em saúde. Essa última variável foi analisada a partir das atas e resoluções do Conselho Municipal de Saúde (Mendonça e col., 2006).

Outras técnicas utilizadas foram: estudo de corte transversal sobre o modelo de gestão e atenção à saúde na atenção básica. Nesse último componente os dados foram obtidos a partir da aplicação de questionários fechados e de entrevistas semiestruturadas com o secretário de saúde do município, coordenador do PSF, coordenador da AB, membros das equipes de saúde da família e gerentes intermediários da atenção básica.

Em ambas as pesquisas foram calculadas amostras aleatórias simples dos profissionais das ESF considerando o número total de ESF em cada um dos quatro distritos sanitários existentes em Manaus. As respostas do questionário fechado foram sistema-

\footnotetext{
2 Documentos consultados: SEMSA. Secretaria Municipal de Saúde de Manaus. Manual do PSF de Manaus. Manaus: Secretaria Municipal de Saúde de Manaus; s/d. SEMSA. Plano de Ação. Programa de Saúde da Família. Manaus: Secretaria Municipal de Saúde de Manaus; 1999. SEMSA. Relatório de Gestão. Manaus: Secretaria Municipal de Saúde de Manaus; 2005. MANAUS. Prefeitura Municipal. Lei Municipal n. 590 de 13 de março de 2001.
} 
tizadas em Planilhas Excel, gerando banco de dados, mediante dupla digitação, transporte subsequente para o programa $S A S$, possibilitando a geração de estatísticas descritivas (Mendonça e col., 2006).

Os marcos referenciais foram similares para as duas pesquisas, caracterizando-se como investigação avaliativa, entendida por Contandriopoulos e colaboradores como aquela que "pretende analisar a pertinência, os fundamentos teóricos, a produtividade, os efeitos e o rendimento de uma intervenção, assim como as relações existentes entre a intervenção e o contexto no qual se situa" (1997, p. 37). Por se tratar de uma pesquisa de base nacional, da qual a investigação feita em Manaus representa apenas um segmento, o modelo teóricológico que a orientou foi único para todo o Brasil, e está descrito em Bodstein e colaboradores (2006). As dimensões contidas no modelo lógico utilizado e que operaram como categorias de análise foram: a) Político-institucional: projeto de governo, capacidade de governo, governabilidade e prioridade da Atenção Básica; b) Organização da atenção: práticas de gestão, práticas na oferta de serviços, sustentabilidade e experiências inovadoras; c) Cuidado integral: acolhimento, vínculo, qualidade técnica e científica, condições e práticas de trabalho, trabalho em equipe; d) Desempenho dos sistemas de saúde: cobertura, cumprimento de metas pactuadas, exercício do controle social e uso da informação para a gestão participativa (Bodstein e col., 2006; Minayo, 2006; Silva e Formigli, 1994). Neste artigo limitarnos-emos à análise das dimensões “a”, "b” e parte da dimensão "d" do supracitado modelo lógico.

Ambas as pesquisas foram submetidas ao Comitê de Ética em Pesquisa da Fundação Oswaldo Cruz, sendo os Termos de Consentimento Livre e Esclarecido elaborados de acordo com a Resolução 196/96 MS e devidamente assinados pelos respondentes.

Os dados foram disponibilizados pela Secretaria de Saúde, de acordo com o solicitado pelos pesquisadores. A confiabilidade das informações secundárias disponíveis foi considerada entre média e boa, porém os documentos acessados continham lacunas que dificultaram a análise.

\section{Apresentação e Discussão dos Resultados}

A operacionalização do PSF em Manaus deu-se com a instalação das Casas de Saúde da Família em 1999 e 2000, de forma acelerada, inauguradas numa média de três casas por semana, no período de implantação do programa; ao todo foram construídas 160 casas para 165 equipes multiprofissionais. Os documentos de criação do programa mostram que seu objetivo inicial foi estender a cobertura da atenção em saúde, por meio das ações básicas restritas, tendo como alvo as famílias residentes em áreas desassistidas. À época, o PMF foi instituído como uma prioridade de gestão; posteriormente as equipes receberam também a missão de operar como uma estratégia de reorientação do modelo assistencial vigente na AB de Manaus (SEMSA, s/d).

Dados da pesquisa avaliativa realizada em 2002 sugerem que a implantação do programa foi produto de uma iniciativa pessoal do prefeito, o qual resistia em aderir às diretrizes nacionais do PSF, por considerá-las muito exigentes frente aos parcos incentivos financeiros propiciados pelo MS (Escorel e col., 2002, p. 44). Em que pese à posição do prefeito, em 2001 o programa foi reestruturado a fim de tornar-se congruente com as normas nacionais de orientação do PSF, facilitar seu reconhecimento pelo MS e fazer jus aos incentivos financeiros do Piso da Atenção Básica (PAB) variável. A necessidade de um melhor relacionamento da Secretaria Municipal de Saúde com o MS foi decisiva para a conversão de PMF em PSF em Manaus, tornando secundária a questão do repasse financeiro (Escorel e col., 2002).

As características de implantação foram marcadas por uma vinculação direta ao gabinete do prefeito e por uma gestão paralela à da própria Secretaria Municipal de Saúde. Quando implantado, o programa contava com espaço físico, orçamento e supervisão próprios, e salários diferenciados quando comparados aos dos profissionais que atuavam em outras UBS. Tais condições geraram repercussões negativas duradouras, que permanecem obstaculizando a integração entre as diversas estratégias de organização da rede básica em Manaus. 
A governabilidade do projeto de implantação do PSF como um tipo de projeto de Governo foi considerada baixa pelos gestores e gerentes entrevistados. Tal condição foi atribuída ao caráter personalista que marcou a instalação e implementação da proposta, levando a um baixo grau de institucionalidade, propiciando uma desvalorização e não priorização do programa, após a saída do dirigente municipal responsável pela iniciativa.

Apesar das muitas afirmações documentais de que a $A B$ representava uma prioridade no planejamento em saúde no município de Manaus, elas não se confirmaram nem no conjunto das ações programadas para o município nem na alocação de recursos humanos. Por exemplo, para o período estudado, as Unidades de Saúde da Família (USF) representavam 70\% de unidades da rede de serviços, mas contavam com apenas $34 \%$ da mão de obra de nível superior alocada no sistema municipal de saúde. Os dados evidenciaram também que, em 2004, do total de repasses do Ministério da Saúde, 28,3\% foram destinados à $\mathrm{AB}$, um percentual mais elevado que os 10,9\% do orçamento municipal para a saúde que foram aplicados em $\mathrm{AB}$.

Os meios de gestão, com seus respectivos instrumentos de planejamento, adotados no sistema municipal de saúde (SMS) de Manaus foram investigados e estão sintetizados no Quadro 1.

\section{Quadro I - Instrumentos de Planejamento do Plano Municipal de Saúde 2003-2005}

\begin{tabular}{|l|l|}
\hline Caracteristicas da Gestão & Município de Manaus \\
\hline Prioridades, metas e estratégias & Claras, objetivas e excessivas \\
\hline $\begin{array}{l}\text { Conhecimento/Incorporação do planejamento por gerentes } \\
\text { locais/unidades }\end{array}$ & Baixo \\
\hline Congruências entre as fontes de informação & Baixa \\
\hline Factibilidade da programação anual & Média \\
\hline Congruência entre o planejado e o executado & Parcial \\
\hline Indicadores/sistema de avaliação e monitoramento & Parcial (indicadores do MS) \\
\hline Autonomia de execução orçamentária pelo SMS & Não \\
\hline Modelo de organização da atenção (PSF versus não PSF) & Paralelismo (PACS - Programa de Agente Comunitário de \\
\hline Grau de informatização das unidades & Saúde -, UBS) \\
\hline Alimentação dos sistemas nacionais de informação & Baixo \\
\hline Comunicação informatizada no SMS (nível central e unidades) & Não \\
\hline Informação subsidia tomada de decisão & Não \\
\hline Regionalização da atenção & Em implantação \\
\hline
\end{tabular}

Fonte: Mendonça e colaboradores, 2006.

Os dados mostram uso incipiente dos instrumentos de planejamento, baixa qualidade técnica na programação instituída, infraestrutura inadequada e qualificação precária e tomada de decisão pouco sustentada por procedimentos racionais de planejamento.

\section{Planejamento situacional com base na família e na comunidade}

De acordo com os dados da pesquisa de 2002, as ESF discutiam semanalmente sua programação de ativi- dades; os médicos seriam os profissionais com maior participação em sua elaboração, seguidos pelos auxiliares de enfermagem, Agentes Comunitários de Saúde (ACS) e enfermeiros (Escorel e col., 2002). Já a investigação realizada em 2006 não mais encontrou ações regulares de programação local, e nem mesmo reuniões de equipe para encaminhamento coletivo dos problemas encontrados. Os profissionais atuavam de modo individualizado, contentando-se com a manutenção de rotinas preestabelecidas nos serviços (Mendonça e col., 2006). Igualmente não 
foram encontradas atividades reconhecidas como inovadoras ou intersetoriais.

Na pesquisa de 2002, a participação da coordenação do PSF nas programações locais era vista pelos profissionais entrevistados como baixa ou insuficiente. Em 2006, a coordenação do programa foi tida como distante ou ausente das atividades desenvolvidas nas unidades, caracterizando uma piora do cenário descrito em 2002. A precarização dos vínculos de trabalho, a insuficiência de mão de obra e a falta de capacitação dos profissionais foram apontadas como parte das causas da não adoção de rotinas de planejamento local.

Nas duas investigações observou-se que o perfil epidemiológico da população atendida era desconhecido, bem como suas demandas e necessidades prioritárias. Em 2002, a USF congregava um grande número de atividades que coexistiam com as ações extramuros. Na investigação mais recente, a observação das ações rotineiras situou a USF como o local quase exclusivo da realização das ações de saúde. A única atividade extramuros encontrada em 2006 foi a visita domiciliar realizada rotineiramente pelos ACS e episodicamente por médicos e enfermeiros. Nos dois momentos, as atividades de assistência individual à demanda espontânea prevaleciam, exceto no caso dos ACS, cujas atividades se voltaram para as famílias de suas áreas de abrangência.

Embora um dos objetivos do PSF seja tomar as famílias como núcleo básico do atendimento à saúde, a trajetória do programa, flagrada por dois momentos de investigação, demonstrou que o indivíduo persistia como foco de atenção nas rotinas das USF.

\section{O PSF de Manaus e a proposta de reorganização da Atenção Básica}

\section{Caráter substitutivo e institucionalização do PSF}

Em 1998, o município de Manaus foi habilitado pela primeira vez na Gestão Plena da Atenção Básica do Sistema Único de Saúde; em 2001, habilitou-se na Gestão Plena da Atenção Básica Ampliada. Embora o processo de habilitação indicasse que o município seria o gestor da rede básica, o governo estadual não repassou as UBS, dificultando a efetivação da municipalização. O processo de transferência iniciou-se somente a partir de 2004, após a abertura de um processo jurídico-administrativo junto ao Ministério Público para tratar do problema. Em consequência, das 23 UBS da gestão estadual, 21 foram repassadas em 2004 e duas permaneceram sob a responsabilidade da Secretaria Estadual de Saúde, tendo sido transformadas em unidades de média complexidade.

Para o período de 2002 a 2005 , de acordo com gestores e gerentes entrevistados, o cenário político da gestão do PSF foi marcado pela disputa de hegemonia entre os governos estaduais e municipais pelo controle da $\mathrm{AB}$. No caso do município de Manaus, uma grande cidade com 1.80o.ooo habitantes, a trajetória de habilitação segundo as Normas Operacionais Básicas evidenciou a persistência do município na condição de Gestão Plena da $\mathrm{AB}$, sem alcançar a Gestão Plena do Sistema Municipal de Saúde. Tal cenário reproduzia, em grande parte, a situação existente nas décadas anteriores, marcada pela forte estadualização do sistema de saúde, inclusive da $A B$, visto que em 2005 a Secretaria Estadual de Saúde ainda prestava $43 \%$ das consultas nesse nível de atenção, na cidade de Manaus.

A conversão do modelo tradicional de $\mathrm{AB}$ para PSF não foi prevista no projeto de implantação da Estratégia Saúde da Família no município de Manaus. Tal situação perdurou até a finalização da coleta de dados da pesquisa de 2006. Em ambas as pesquisas aqui tratadas, não foi encontrada, nas fontes documentais acessadas, a programação de estratégias voltadas para a conversão das UBS previamente existentes em USF. A análise documental do planejamento municipal no período evidenciou persistência, paralelismo e competição entre os modelos de AB convencional e ESF, com programação de atividades e metas específicas para cada um deles, em cada ano investigado, além de ausência de ações de articulação e/ou integração entre as duas formas de atenção. Igualmente não foram encontradas programação de estratégias que favorecessem a descentralização do planejamento e gestão local na rede de USF no município.

Ao paralelismo dos dois tipos de rede aliou-se a implantação, a partir de 2005, dos distritos sanitários no sistema municipal de saúde de Manaus. As fontes documentais investigadas não explicitam com clareza como a distritalização promoveria a integração das duas redes de serviços. Entretanto, 
um trecho do Relatório de Gestão de 2005 (SEMSA, 2005) recomendava efetivar a "implantação das sedes distritais de forma gradativa, objetivando a incorporação das equipes do PMF às unidades básicas de saúde tradicionais e a definição das referências para as especialidades". O teor do documento sugere que as equipes de saúde da família sejam incorporadas ao modelo tradicional de oferta de atenção básica, e não o inverso, tal como preconizado pela proposta do Ministério da Saúde.

Um aspecto importante, ainda que insuficiente para a conversão do modelo, é o desempenho do sistema de saúde, que será aqui analisado através da cobertura da atenção ofertada pelo PSF em Manaus. Embora sua implantação em Manaus tenha ocorrido em 1999, e em 2001 o programa contasse com 171 equipes multiprofissionais atuando (Tabela 1), não houve incremento do número de equipes nos anos subsequentes, ao passo que o crescimento demo- gráfico em Manaus atingiu uma média de 3,73\% ao ano, no período de 2000 a 2007 , com estimativa de crescimento para 2008 e 2009 de $2,28 \%$ a.a $a^{3}$. A baixa capacidade instalada gerou uma defasagem entre a população cadastrada no PSF, ou seja, aquela cuja responsabilidade sanitária é formalmente reconhecida pela rede PSF e aqueles efetivamente atendidos, cujo número ultrapassa a capacidade instalada das unidades e para os quais não existe uma efetiva programação de metas e provisão de insumos.

O sistema também conviveu com a carência de profissionais qualificados para atuar no PSF. 0 gestor entrevistado informou que não foi possível abrir vagas em concurso público realizado no período para profissionais do PSF, devido à insuficiência de especialistas em saúde da família com habilitação para formar um quadro permanente no órgão. Persistiu, portanto, a precarização da força de trabalho, contratada mediante regime temporário de trabalho.

\section{Tabela I - Cobertura do PMF/PSF Manaus, no período de 1999 a 2009}

\begin{tabular}{lccccccccccc} 
Ano & ${ }^{*} 1999$ & ${ }^{*} 2000$ & ${ }^{*} 2001$ & 2002 & 2003 & 2004 & 2005 & 2006 & 2007 & 2008 & 2009 \\
\hline$N^{\circ}$ Equipes & 106 & 166 & 171 & 82 a 150 & 173 & 173 & 173 & 125 a 180 & 150 a 180 & 167 a 177 & 167 \\
Cobertura & 33,27 & 45,84 & 46,88 & $19,48 \mathrm{a}$ & $35,64 \mathrm{a}$ & $39,08 \mathrm{a}$ & 39,08 & 26,16 a & $30,58 \mathrm{a}$ & $34,05 \mathrm{a}$ & 33,71 \\
$\%$ & & & & 35,40 & 40,63 & 40,09 & & 40,66 & 36,70 & 36,09 & \\
\hline
\end{tabular}

Fontes: Escorel e colaboradores, 2002; Mendonça e colaboradores, 2006.

Brasil, 2009 (Disponível em: http://dtr2004.saude.gov.br/dab/historico_cobertura_sf.php).

A tabela 1 mostra que a cobertura do programa oscila significativamente no decorrer dos anos, mas mantendo uma tendência de declínio. Apesar da acelerada expansão inicial de cobertura, com implantação de grande número de equipes no período de 1999 a 2001, em Manaus o PSF nunca atingiu 50\% de cobertura da população-alvo, mantendo-se a tendência de declínio nos anos recentes. Numa série histórica de 1998 a 2001, observou-se que o programa contribuiu significativamente para o crescimento da rede ambulatorial da cidade, que saltou de 273 unidades para 437. Em 2001, o número de USF correspondia a 44,6\% da rede ambulatorial do SUS sob a gestão municipal (Escorel e col., 2002). Destaca-se que os dados brutos de cobertura escondem as oscilações geradas pela alta rotatividade de profissionais. Ao longo do período analisado, foram poucos os momentos em que as unidades dispuseram de "equipe cheia", ou seja, que contavam com a totalidade de integrantes previstos nas normas do prog rama.

Para avaliar a institucionalização da Estratégia de Saúde da Família no país, Mendes (2002) propôs uma escala de quatro estágios: i) Transição inicial cobertura de 10 a $25 \%$ e instabilidade institucional; ii) Transição intermediária - cobertura de 25 a $50 \%$ e baixa institucionalidade; iii) Transição terminal - cobertura de 50 a 70\% e institucionalização nos serviços de saúde (posição da APS na rede de serviços), nos espaços educacional (formação dos profissionais), corporativo (organização dos pro- 
fissionais de APS) e de representação populacional (saúde da família como valor societal); iv) Momento de consolidação - cobertura acima de $70 \%$ e institucionalização nos cinco espaços, ainda que de forma assimétrica (Mendes, 2002, p. 31-34).

Se considerarmos os critérios estabelecidos por Mendes (2002) para definir o grau de implantação do PSF em Manaus, é possível enquadrá-lo em momento de transição intermediária situando-se na faixa de cobertura de 25 a 50\%, com baixa institucionalização, ainda distante da consolidação.

Nessa mesma ordem de questões, um entrave de grandes proporções ao PSF em Manaus é a não implantação de sistema de referência e contrarreferência, mesmo após sete anos de existência do programa. O mesmo problema já foi identificado em outros locais, nos quais se observou que a implantação da estratégia saúde da família gerou incremento de demanda para os níveis de maior complexidade, que não se organizaram para incorporar a pressão de demanda produzida pela expansão da cobertura do nível primário, gerando filas de espera pelo atendimento (Teixeira 2003/2004). Além disso, tal como em outras realidades (Franco e Merhy, 2003), os gestores e gerentes entrevistados reconheceram que a falta de atendimento de urgência nas USF acarreta baixa credibilidade de uma estratégia como a da Saúde da Família.

Os três primeiros anos do PSF foram marcados pela valorização de ações de educação em saúde nos domicílios, passando posteriormente a priorizar a assistência fornecida no interior das unidades, parcialmente voltada para a demanda programada, tipo atenção programática. Nessas circunstâncias, a baixa resolutividade das USF levava os ACS a encaminhar a demanda espontânea e/ou em situações de emergência para outros serviços, gerando forte insatisfação entre profissionais e usuários, que não viam vantagem na vinculação a uma USF (Mendonça e col., 2006).

\section{Territorialização}

A implantação dos distritos sanitários em Manaus com sua respectiva territorialização iniciou-se apenas em 2005, ainda que tivessem sido criados em março de 2001, por meio da Lei n. 590 (Manaus, 2001). Tal iniciativa também implicou em reestruturação das áreas de cobertura do PSF na cidade. Nas atuais condições de organização de serviços, a investigação demonstrou que as áreas de abrangência das USF, localmente subdivididas nas chamadas "microáreas", não obedecem aos critérios habituais de territorialização em saúde. De acordo com os entrevistados, na implantação do PSF sua delimitação se deu apenas em função do conhecimento que os ACS detinham dos arredores das unidades em que trabalhavam. Após um recenseamento domiciliar feito por eles, definiu-se uma densidade populacional máxima por microárea, a ser acompanhada por um ACS, caracterizando-se desse modo a territorialização de cada USF. Destaca-se que a territorialização levada a cabo pelo programa não guarda congruência com outras formas oficiais de territorialização, como a delimitação por setores censitários praticada pelo Instituto Brasileiro de Geografia e Estatística (IBGE), o que dificulta o acesso aos indicadores socioeconômicos que expressem as condições de vida dessas populações e prejudica o planejamento local.

Isso, porém, não parece ter dificultado certos benefícios da territorialização, tais como o estabelecimento de vínculo entre a população e os serviços de saúde. Já em 2002, 95\% das pessoas entrevistadas por pesquisadores que investigavam a implantação do PSF em Manaus (Escorel e col., 2002) referiam conhecer o profissional que lhes assistia; o mesmo percentual de respondentes considerou-o atencioso; 90\% deles informaram que alguém da família já havia sido assistido por algum profissional da ESF. Nessas respostas, o ACS se destacou tanto por ser conhecido quanto por conhecer bem os problemas de saúde da população atendida, e por manter um bom relacionamento com as famílias sob seus cuidados. Se considerarmos que o primeiro requisito para o estabelecimento de vínculo é o conhecimento entre as pessoas atendidas, os dados da pesquisa indicam que esse laço foi firmado no início da operacionalização do programa. Na pesquisa de 2006, os usuários mantiveram sua opinião satisfatória quanto ao atendimento dos ACS, mas expressaram queixas frente à recorrente falta de profissionais de saúde de nível superior, medicamentos e exames complementares de diagnóstico nas unidades. 


\section{Integração com instituições e organizações sociais}

A integração com as instituições e organizações sociais é recomendada na Política Nacional da Atenção Básica (Brasil, 2006) como forma de suprir globalmente as necessidades de saúde dos usuários que demandam as USF. Sobre tal aspecto, a análise dos documentos pesquisados em Manaus mostrou que as propostas de integração das USF com outras instituições e campos de ação (intersetoriais) em sua área de abrangência não surgem como prioridade de ação, no período estudado. A observação da rotina dos serviços também indicou a inexistência de integração nos diversos níveis do sistema da saúde e a penalização dos usuários, que enfrentam longas filas quando necessitam de atendimento em unidades de complexidade maior que aquela instalada na AB (Souza e Garnelo, 2008).

A referência para os serviços especializados era dificultada pela divisão institucional de tarefas entre instituições municipais (responsáveis pela AB) e instituições estaduais (responsáveis pela rede especializada), cujas disputas políticas têm obstaculizado a elaboração de um planejamento que articule e integre demandas e programações de saúde para o sistema como um todo, penalizando ainda mais o usuário.

\section{Participação popular e controle social}

As propostas de reorganização da atenção básica em Manaus começaram a ser discutidas no Conselho Municipal de Saúde (CMS) em 1991, ocasião em que o Plano Municipal de Saúde (PMS) já apontava para o PSF como a porta de entrada do sistema de saúde. Apesar da recomendação, as premissas iniciais não foram viabilizadas, tendo-se, em seu lugar, implantado o PMF.

De acordo com os documentos consultados, o exercício do controle social sobre o PSF deu-se ao longo dos anos pelo CMS, que acompanhou o cumprimento das ações previstas no PMS e empreendeu diversas visitas às USF. Da mesma forma, os Relatórios de Gestão e os relatórios de visitas de conselheiros, para o esclarecimento de denúncias envolvendo o programa, também teriam auxiliado no controle social. A partir do ano de 2004, o coordenador do PSF passou a ocupar um dos assentos do CMS, viabilizando uma maior aproximação com o controle social. Com a extinção do cargo, em 2005, essa representação passou a ser feita pelos coordenadores dos distritos sanitários, pressupondo-se que, estando o PSF inserido nos mesmos, sua representatividade estaria efetivada.

As pesquisas mostraram que, por ocasião da implantação do programa, apenas o cadastramento da população adscrita foi efetivado e que as ações de saúde eram desenvolvidas com base nas ações programáticas por ciclo de vida e agravos prevalentes. A população não participou da elaboração do diagnóstico de saúde da comunidade e do planejamento local, o que obstou seu entendimento sobre o processo saúde-doença do território e o exercício da cidadania. Além disso, a ausência de ações regulares de educação em saúde nas USF inviabilizou a capacitação dos usuários para a adoção de estratégias de promoção da saúde, além do exercício qualificado do controle social.

Um dos objetivos definidos para o PSF é o de "estimular a organização da comunidade para o efetivo exercício do controle social”. As formas prioritárias desse exercício, expressas na Constituição Federal de 1988 e na Lei Orgânica do SUS n. 8142/9o, são as conferências de saúde e os conselhos de saúde. Apesar dessas recom endações oficiais e da avaliação positiva que o programa obteve junto ao CMS de Manaus, a pesquisa não encontrou prova documental ou depoimentos que corroborassem a ideia de que as ações do PSF tenham contribuído para incrementar a participação social nos locais em que atua.

\section{Conclusão}

Extensão de cobertura ou reorganização da Atenção Básica? A Estratégia Saúde da Família foi inicialmente proposta para Manaus como Programa Saúde da Família nos moldes concebidos pelo MS. Abortada essa modalidade de implantação, surgiu em seu lugar o Programa Médico da Família, cuja caracterização inicial já o descredenciava como proposta de mudança, uma vez que praticava um modelo médico-centrado, voltado para o atendimento curativo de indivíduos doentes, em detrimento do modelo de atenção voltado para usuários e coletividades. Suas principais conquistas foram a expansão de cobertura da $\mathrm{AB}$, com ampliação do acesso a ela e da força 
de trabalho ali alocada, bem como a valorização do programa pelos usuários.

Entretanto, os dados aqui trabalhados mostraram que o PSF não adquiriu um caráter substitutivo das UBS antecedentes à sua implantação e que permaneceu como um sistema paralelo na rede de $A B$ em Manaus, mostrando-se, portanto, incapaz de induzir mudanças nos processos de trabalho nesse nível de atenção. A territorialização foi empreendida, mas de modo incompleto, restringindo-se às áreas ao redor das USF, sem impactar efetivamente as estratégias de territorialização do sistema municipal de saúde como um todo. O planejamento situacional tendo como base as necessidades das famílias e comunidades foi inicialmente implantado, mas refluiu nos anos subsequentes, até cessar completamente. A intersetorialidade, expressa mediante a integração com outras instituições e organizações sociais, não se consolidou nos anos que sucederam a implantação do programa. Não se evidenciou a presença de participação popular na gestão local das USF. Nas instâncias mais amplas de controle social, como o Conselho Municipal de Saúde, evidenciou-se baixa permeabilidade aos temas de interesse do PSF. A documentação acessada não demonstrou presença significativa de informações geradas em nível local para a tomada de decisão nos fóruns maiores de controle social.

O programa teve, no período de 1999 a 2001, sua fase de expansão, num ritmo acelerado, o que inviabilizou uma melhor qualificação das ESF. Ainda que o PSF seja citado, em diversos documentos do SMS de Manaus, como uma estratégia estruturante da $\mathrm{AB}$ com vistas à reorganização do modelo assistencial, a análise mostrou que ele se instituiu como um programa isolado. Alcançou relativo sucesso na extensão de cobertura, mas essa trajetória não foi mantida, instalando-se uma tendência de queda nos anos subsequentes.

A isso se associaram outros problemas, como a baixa resolutividade das ações desenvolvidas nas USF e a impossibilidade de operacionalizar a referência para os níveis de maior complexidade; a manutenção da prioridade ao atendimento curativo dos indivíduos, em detrimento dos cuidados ofertados às famílias e à comunidade; e a não prioridade do PSF na agenda política do município após o período de implantação.

Aos entraves e às limitações regionais do programa agregam-se os problemas estruturais do PSF, dentre os quais se destaca a obrigatoriedade do formato único nacional, incapaz de enfrentar a diversidade das condições sociais existentes no país. Além disso, a mudança do modelo de assistência hegemônico tende a enfrentar barreiras, dada a resistência dos profissionais afetos ao antigo modelo e dos usuários, que tendem a preferir o atendimento especializado, apesar dos limites de oferta enfrentados no SUS.

Os desafios que se colocam à estratégia de mudança do modelo de $\mathrm{AB}$ são muitos. A superposição de dois modelos, que há muitos anos persiste em Manaus, pode ser tomada como um tempo limite de transição. Vislumbra-se uma retomada da perspectiva de mudanças com a implantação dos distritos sanitários, no final de 2007, os quais têm a potencialidade de contribuir, efetivamente, para a almejada reorganização do modelo de $\mathrm{AB}$ e conformar uma rede adequadamente integrada.

\section{Referências}

BODSTEIN, R. et al. Estudos de Linha de Base do Projeto de Expansão e Consolidação do Saúde da Família (ELB/Proesf): considerações sobre seu acompanhamento. Ciência e Saúde Coletiva, Rio de Janeiro, v. 11, n. 3, p. 725-731, 2006.

BRASIL. Ministério da Saúde. Saúde da família: uma estratégia para a reorientação do modelo assistencial. Brasília, DF, 1997.

BRASIL. Ministério da Saúde. Secretaria de Assistência Saúde. Coordenação de Saúde da Comunidade. Manual do sistema de informação de atenção básica. Brasília, DF, 2003.

BRASIL. Ministério da Saúde. Secretaria de Atenção à Saúde. Departamento de Atenção Básica. Política Nacional de Atenção Básica. Brasília, DF, 2006.

BRASIL. Ministério da Saúde. Departamento de Atenção Básica. Evolução do credenciamento e implantação da estratégia saúde da família. Brasília. 2009. Disponível em: www.saude.gov.br/ dab. Acesso em: 3 nov. 2009. 
CAMPOS, G. W. S. Debate sobre o artigo de Conill. Cadernos de Saúde Pública, Rio de Janeiro, v. 24, p. S17-S19, 2008. Sup1emento 1.

CONILL, E. M. Ensaio histórico-conceitual sobre a atenção Primária à Saúde: desafios para a organização de serviços básicos e da Estratégia Saúde da Família em centros urbanos no Brasil. Cadernos de Saúde Pública, Rio de Janeiro, v. 24, p. S7-S16, 2008. Suplemento 1.

CONTANDRIOPOULOS, A. et al. Avaliação na área da saúde conceitos e métodos. In: HARTZ, Z. M. (Org.). Avaliação em saúde: dos modelos conceituais a práticas da implantação de programas. Rio de Janeiro: Fiocruz, 1997. p 29-47.

ESCOREL, S. et al. Avaliação da implementação do Programa Saúde da Família em grandes centros urbanos: relatório final de Manaus-AM. Brasília, DF: Ministério da Saúde, 2002.

ESCOREL, S. et al. O Programa de Saúde da Família e a construção de um novo modelo para a atenção básica no Brasil. Revista Panamericana de Salud Publica, Washington, DC, v. 2, n. 2, p.164176, 2007. Disponível em <http://www.scielosp. org/pdf/rpsp/v21n2-3/11.pdf>. Acesso em: 15 mar. 2008.

FRANCO, T. B.; MERHY, E. E. PSF: contradições de um programa destinado à mudança do modelo tecnoassistencial. In: MERHY, E. E. et al. $O$ trabalho em saúde: olhando e experenciando o SUS no cotidiano. São Paulo: Hucitec, 2003. p. 55-124.

GARBOIS, J. A.; VARGAS, L. A.; CUNHA, T. S. O direito à saúde na Estratégia Saúde da Família: uma reflexão necessária. Physis: Revista de Saúde Coletiva, Rio de Janeiro, v. 18, n. 1, p. 27-44, 2008.

GIOVANELLA, L. Atenção primária à saúde seletiva ou abrangente? Cadernos de Saúde Pública, Rio de Janeiro, v. 24, p. 21-23, 2008. Suplemento1.

MENDES, E. V. A atenção primária á saúde no SUS. Fortaleza: Escola de Saúde Pública do Ceará, 2002.

MENDONÇA, M. H. M. et al. Estudos de linha de base nos municípios selecionados para o componente III do Proesf Lote Norte 1 - relatório final de Manaus -AM. Brasília, DF: Ministério da Saúde, 2006.
MERHY, E. E.; FRANCO, T. B. Programa Saúde da Família: somos contra ou a favor? Saúde em Debate, Rio de Janeiro, v. 26, n. 6o, p. 118-122, 2002. MINAYO, M. C. S. Pesquisa por triangulação de métodos. In: BOSI, M. L. M.; MERCADO, F. J. (Ed.). Avaliação qualitativa de programas de saúde: enfoques emergentes. Petrópolis: Vozes, 2006. p. 163-19o.

OPAS/OMS - Organização Panamericana da Saúde/Organização Mundial da Saúde. Renovação da Atenção Primária em Saúde nas Américas. Documento de posicionamento da Organização Pan-Americana de Saúde/OMS. Disponível em: http://www.opas.org.br/servico/temas documento_detalhe.cfm?CodSubTema $=147 \&$ CodEs pecifico $=5575$. Acesso em: 10 dez. 2008.

ROSA, W. A G. A.; LABATE, R. C. Programa Saúde da Família: a construção de um novo modelo de assistência. Revista Latino-Americana de Enfermagem, São Paulo, v. 13, n. 6, p. 1027-1034, 2005 .

SILVA, L. M. V.; FORMIGLI, V. L. A. Avaliação em saúde: limites e perspectivas. Cadernos de Saúde Pública, Rio de Janeiro, v. 10, n. 1, p. 80-91, 1994.

SOARES, L. As atuais políticas de saúde: os riscos do desmonte neoliberal. Revista Brasileira de Enfermagem, Brasília, DF, v. 53, p. 17-24, 2000. Número especial.

SOUSA, M. F.; HAMANN, E. M. Programa Saúde da Família no Brasil: uma agenda incompleta? Ciência e Saúde Coletiva, Rio de Janeiro, v. 14, p. 1325-1335, 2009. Suplemento 1.

SOUZA, H. Saúde da família: desafios e conquistas. In: NEGRI, B.; VIANA, A. L. D. (Org.). $O$ Sistema Único de Saúde em dez anos de desafio. São Paulo: Sobravime;Celag, 2002. p. 221-240.

SOUZA, M. P. L.; GARNELO, L. É muito dificultoso!: etnografia dos cuidados a pacientes com hipertensão e/ou diabetes na Atenção Básica em Manaus. Cadernos de Saúde Pública, Rio de Janeiro, v. 24, p. 591-599, 2008. Suplemento 1.

TEIXEIRA, C. F. A mudança no modelo de atenção à saúde no SUS: desatando nós, criando laços. Saúde em Debate, Rio de Janeiro, v. 27, n. 65, p. 257-277, 2003. 
TEIXEIRA, C. F. Saúde da família, promoção e vigilância: construindo a integralidade da atenção á saúde no SUS. Revista Brasileira de Saúde da Família, Brasília, DF, ano V, n. 7, p. 10-23, 2003/2004.

TRAD, L. A. B.; BASTOS, A. C. S. O impacto sóciocultural do Programa de Saúde da Família (PSF): uma proposta de avaliação. Cadernos de Saúde Publica, Rio de Janeiro, v. 14, n. 2, p. 429-435, 1998. VIANA, A. L. D.; DAL POZ, M. R. Reforma em saúde no Brasil: Programa de Saúde da Família: Physis: Revista de Saúde Coletiva, Rio de Janeiro, v. 15, p. 225-264, 2005. Suplemento1.

Recebido em: 19/05/2009

Reapresentado em: 22/12/2009

Aprovado em: 04/02/2010

604 Saúde Soc. São Paulo, v.19, n.3, p.592-604, 2010 